\title{
Exclusive Photoproduction at HERA
}

\author{
Dorota Szuba*i \\ DESY, Notkestrasse 85, D-22607 Hamburg, Germany; \\ on leave INP, ul.Radzikowskiego 152, 31-342 Cracow, Poland \\ E-mail: dorota.szuba@desy.de
}

The exclusive $\Upsilon$ photoproduction, $\gamma p \rightarrow \Upsilon p$, has been studied with the ZEUS experiment in $e p$ collisions at HERA using an integrated luminosity of $468 \mathrm{pb}^{-1}$. The measurement covers the kinematic range $60<W<220 \mathrm{GeV}$ and $Q^{2}<1 \mathrm{GeV}^{2}$, where $W$ is the photon-proton centre-ofmass energy and $Q^{2}$ is the photon virtuality. These results, which represent the analysis of the full ZEUS data sample for this channel, are compared to predictions based on perturbative QCD. The proton-dissociative diffractive photoproduction of $J / \psi$ mesons, $\gamma p \rightarrow J / \psi Y$, with $Y$ being the proton dissociative system, has been studied in ep collisions with the ZEUS detector at HERA using an integrated luminosity of $112 \mathrm{pb}^{-1}$ in the range $30<W<160 \mathrm{GeV}$ and $2<|t|<30 \mathrm{GeV}^{2}$, where $t$ is the four-momentum transfer squared at the proton vertex. The cross section is presented as a function of $W$ and $t$. The results are compared to perturbative QCD calculations. The first measurement of diffractive scattering of quasi-real photons with large momentum transfer $\gamma p \rightarrow \gamma Y$ was made using the $\mathrm{H} 1$ detector at HERA. The measurement is performed for initial photon virtualities $Q^{2}<0.01 \mathrm{GeV}^{2}$. Cross sections are measured as a function of $W$ and $t$ in the range $175<W<247 \mathrm{GeV}$ and $4<|t|<36 \mathrm{GeV}^{2}$. The data are compared to a model based on perturbative QCD using a leading-logarithmic approximation of the BFKL evolution. Based on data collected with the H1 detector at HERA in 2005, cross sections for elastic $\rho$ photoproduction have been measured at $|t|<0.58 \mathrm{GeV}^{2}$ and $8<W<94 \mathrm{GeV}$. This data has been combined with cross sections published previously by the Omega and ZEUS collaborations in a global fit to determine the Pomeron trajectory $\alpha(t)$ in 13 bins of $t$ from the $W$-dependence of the elastic $\rho$ production cross section.

European Physical Society Europhysics Conference on High Energy Physics, EPS-HEP 2009, July 16 - 222009

Krakow, Poland

\footnotetext{
${ }^{*}$ Speaker.

${ }^{\dagger}$ on behalf of the ZEUS and H1 Collaborations
} 


\section{Introduction}

The H1 and ZEUS experiments at the HERA electron-proton collider have provided extensive studies of vector meson (VM) production [1]. The HERA data cover wide kinematic region, in which perturbative QCD (pQCD) is expected to be applicable, as well as regions in which no hard scale is present and non-perturbative processes dominate. Hence the diffractive production of vector mesons gives the opportunity to explore the partonic nature of the diffractive exchange and to investigate the transition region between soft physics, already explored by fixed target experiments, and the hard, QCD physics.

The production of vector mesons like the $\rho^{0}, \omega, \phi, J / \psi, \psi^{\prime}, \Upsilon$ or scattered photons $\gamma$ has been studied in the diffractive process $e p \rightarrow e V M / \gamma Y$, with $Y$ being either a proton (elastic process) or a proton remnant system (proton-dissociative process). The characteristic variables of such processes are: the mass of the vector meson, $m_{V M}$, the photon-proton centre-of-mass energy, $W$, the photon virtuality, $Q^{2}$, and the four-momentum transfer squared at the proton vertex, $|t|$. At high energies, vector meson production can be modelled as in the proton rest frame, in which the incoming electron emits a virtual photon, which subsequently fluctuates into a $q \bar{q}$. Next, the $q \bar{q}$ pair forms a vector meson. All these steps are well separated in time and the cross section can be factorized using the dipole cross section on the proton [2]. The transverse size of the $q \bar{q}$ pair controls the character of the interaction. If the transverse momentum is small, the quarks form a colour dipole, whose transverse size is large. The interaction with the proton looks then similar to soft hadron-hadron scattering. When the transverse size of the colour dipole is small the interaction with the proton can be calculated perturbatively.

The non-perturbative approach, based on Regge phenomenology [3] and the Vector Dominance Model (VDM) gives a successful description of light vector mesons $(\rho, \omega, \phi)$ production at low values of $Q^{2}$ and $|t|$. In this approach the diffractive interaction is mediated by the exchange of the vacuum quantum numbers which corresponds to soft Pomeron exchange. This approach predicts: weak energy dependence of the cross section $\left(\sigma \propto W^{0.22}\right)$, shrinkage of the diffractive peak with increasing $W\left(d \sigma / d t \propto e^{-b t}\left(W / W_{0}\right)^{4\left(\alpha_{I P}(t)-1\right)}\right.$, with $\alpha_{I P}(t)=\alpha_{I P}(0)+\alpha_{I P}^{\prime} t$ and $b=b_{0}+4 \alpha^{\prime} \ln \left(W / W_{0}\right)$ ), S-Channel Helicity Conservation, SCHC, (the VM retains the helicity of the photon).

For large values of $Q^{2}, m_{V M}$ or $|t|$ the process is expected to be 'hard' and hence pQCD should be applicable. In this approach the colour dipole interacts with the proton (or a single parton in it) by the exchange of a colour singlet state, which is a two-gluon system or a gluon ladder (the latter is often called the hard or $\mathrm{pQCD}$ pomeron). The $q \bar{q}$ scattering off the proton is described by pQCD, while the transitions $\gamma \rightarrow q \bar{q}$ and $q \bar{q} \rightarrow V M$ are modelled with the respective wave functions. The cross section is proportional to the square of the gluon density $g\left(x, Q^{2}\right)$ in the proton [2]: $\sigma \propto \alpha_{s}^{2}\left(Q^{2}\right) / Q^{6}\left[x g\left(x, Q^{2}\right)\right]^{2}$, with $x \approx Q^{2} / W^{2}$ (valid for small $x$ ). A steeper rise of the cross section with $W$ is predicted, along with a weaker $t$ dependence of the cross section and smaller shrinkage with increasing $W$, dominant longitudinal $\gamma^{*}$ polarization, possible SCHC violation. At larger $|t|$ values $\left(|t|>1 \mathrm{GeV}^{2}\right)$ the proton-dissociation process dominates. In this region the cross section is expected to have a power-law decrease with $|t|$. 

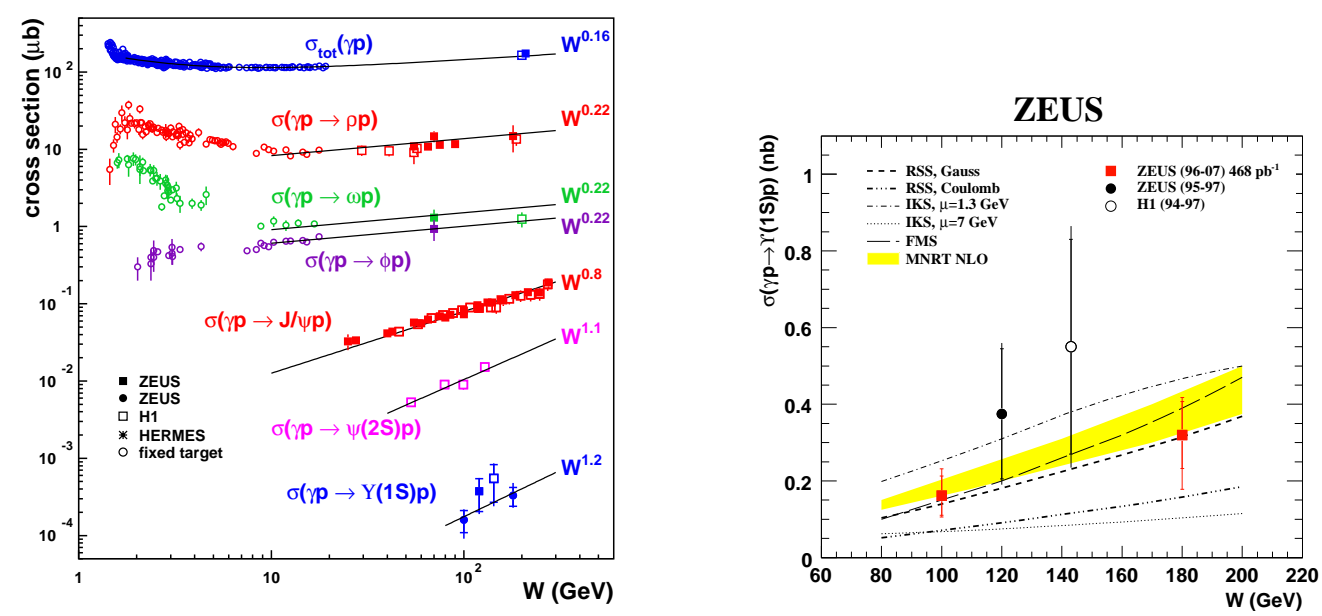

Figure 1: The total photoproduction cross section and the cross sections for exclusive vector meson production as a function of $W$. The lines show a $W^{\delta}$ dependence with the corresponding $\delta$ values indicated (left). The cross section of exclusive $\Upsilon$ photoproduction as a function of $W$ (right).

\section{2. $\Upsilon$ photoproduction}

The energy dependence of the cross section for exclusive photoproduction of vector mesons at HERA, $\sigma_{\gamma p \rightarrow V M p}$, has the form $\sigma \propto W^{\delta}$. For the light vector mesons as well as for the total cross section $\delta=0.22$ as predicted by Regge phenomenology. In the case of heavy vector mesons, where the mass provides a large scale, the $W$ dependence is much steeper: $\delta=0.7$ for $J / \psi$ rising to 1.2 for $\Upsilon$, as shown in Fig. 1(left). This is understood in terms of the rise of the gluon density at low $x$.

The exclusive photoproduction of $\Upsilon(1 S)$ mesons [4] has been studied with the ZEUS detector in the kinematic range $60<W<220 \mathrm{GeV}, Q^{2}<1 \mathrm{GeV}^{2}$ and $\sqrt{s}=318 \mathrm{GeV}$ using the muon decay channel. Fig. 1 (right) shows the extracted cross section for the two independent $W$ ranges, $60<W<130 \mathrm{GeV}$ and $130<W<220 \mathrm{GeV}$. Data were compared to previous ZEUS result [5] based on a partially overlapping data set and the $\mathrm{H} 1$ result [6]. A fit to the form $W^{\delta}$ provided $\delta=1.2 \pm 0.8$, a value consistent with the theoretical expectation [5]. The data were compared to several theoretical models. The FMS [7], IKS [8], MNRT [9] and RSS [10] calculations are consistent with the data. In the IKS case, a calculation using an intermediate scale is preferred. In the RSS case, the data seem to favour a Gaussian-like wave function.

\section{High $|\mathbf{t}|$}

The high-It| domain is dominated by the processes in which the scattered proton dissociates into a hadronic final state. One of such processes, the proton-dissociative $J / \psi$ photoproduction, $\gamma p \rightarrow J / \psi Y$, where $J / \psi \rightarrow \mu^{+} \mu^{-}$, was studied with the ZEUS detector [11]. The considered kinematic region was $30<W<160 \mathrm{GeV}, 2<|t|<20 \mathrm{GeV}^{2}$ and $z>0.95$, where $z$ is defined as $z=(P \cdot v) /(P \cdot q)$ and $P, v, q$ are the four-momenta of the incident proton, produced vector meson and exchanged photon, respectively. 

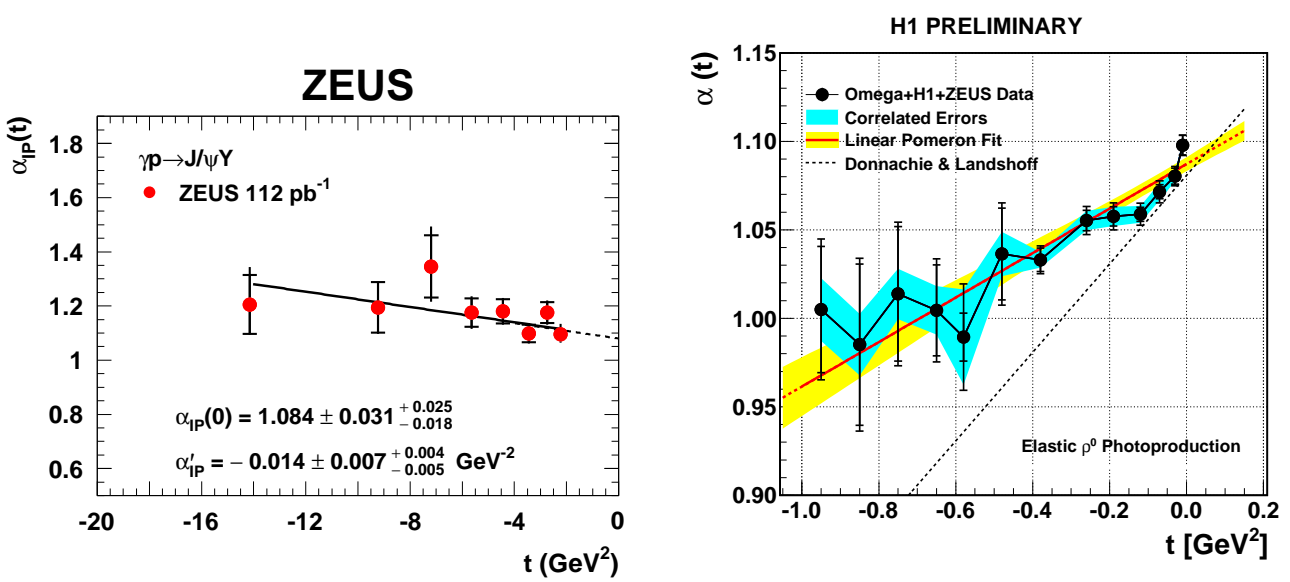

Figure 2: The Pomeron trajectory as a function of $t$ for proton-dissociative $J / \psi$ photoproduction, $\gamma p \rightarrow$ $J / \psi Y$ (left) and for elastic $\rho$ photoproduction, $\gamma p \rightarrow \rho p$ (right).

The $|t|$ dependence of the differential cross section, $d \sigma / d|t|$, falls steeply with $|t|$. The data behave approximately power-like, $\sim|t|^{-n}$, with the power $n$ increasing with $|t|$ but cannot be described by a single power-law dependence in the whole $|t|$ region.

The $W$ dependence of the differential cross-section was measured for eight fixed $t$ values and in each $t$ bin parameterized as $\sigma \sim W^{\delta}$. The effective Pomeron trajectory was derived from a measurement of the $W$ dependence through the relation $\alpha_{I P}=(\delta+4) / 4$ and was parameterized by a linear fit to the form $\alpha_{I P}(t)=\alpha_{I P}(0)+\alpha_{I P}^{\prime} \cdot t$, as shown in Fig. 2 (left). The fit yields an intercept $\alpha_{I P}(0)=$ $1.084 \pm 0.031$ (stat.) ${ }_{-0.018}^{+0.025}$ (syst.) and a slope $\alpha_{I P}^{\prime}=-0.014 \pm 0.007$ (stat. $)_{-0.005}^{+0.004}$ (syst.) $\mathrm{GeV}^{-2}$. The value of the intercept is consistent with that of the so-called "soft" Pomeron [12] (1.0808). The slope is consistent with the predictions of the BFKL Pomeron $[13,14]$ but different from that of the "soft" Pomeron [15] $\left(0.25 \mathrm{GeV}^{-2}\right)$ and also different from the results of the measurement of elastic $\rho$ phototoproduction [20] which yields $\alpha_{\mathbb{P}}^{\prime}=0.126 \pm 0.013 \pm 0.012 \mathrm{GeV}^{-2}$, see Fig. 2 (right).

The $t$ and $W$ dependence of the cross section were compared to different theoretical predictions, as shown in Fig. 3. The DGLAP-motivated GLMN LL [16] calculation (model valid to $|t|<10 \mathrm{GeV}^{2}$ ) can describe the behaviour of the data, both in $t$ and in $W$, up to $|t|=5 \mathrm{GeV}^{2}$. The BFKL-motivated EMP LL [17] calculation fails to describe the data in the kinematic region of the present measurement. The FSZ $[18,19]$ calculation describes the $t$ dependence of the cross section only up to $|t|=12 \mathrm{GeV}^{2}$ and fails to reproduce the $W$ dependence.

The first measurement of diffractive scattering of quasi-real photons at large $|t|[21], \gamma p \rightarrow \gamma Y$, was done with the $\mathrm{H} 1$ detector. This process was studied in the range $175<W<247 \mathrm{GeV}$, $4<|t|<36 \mathrm{GeV}^{2}, Q^{2}<0.01 \mathrm{GeV}^{2}$ and $y_{I P}<0.05$, where $y_{I P}=P \cdot(q-v) /(P \cdot q)$. The cross section as a function of $W$ is shown in Fig. 4 (left). The cross section was fitted to the form $\sigma \sim W^{\delta}$ and the fit yields $\delta=2.73 \pm 1.02$ (stat. $)_{-0.78}^{+0.56}$ (syst.). The differential cross-section, $d \sigma / d t$, at $W=219 \mathrm{GeV}$ is shown in Fig. 4 (right) together with the fit to the form of $d \sigma / d t \sim|t|^{-n}$, where $n=2.60 \pm 0.19$ (stat. $)_{-0.08}^{+0.03}$ (syst.). The cross sections were compared to the LLA BFKL predictions [22, 23, 24], using the HERWIG Monte Carlo with no $|t|$ reweighting, as shown in Fig. 4. The predictions were normalized to the integrated measured cross section. In the asymptotic 


\section{ZEUS}

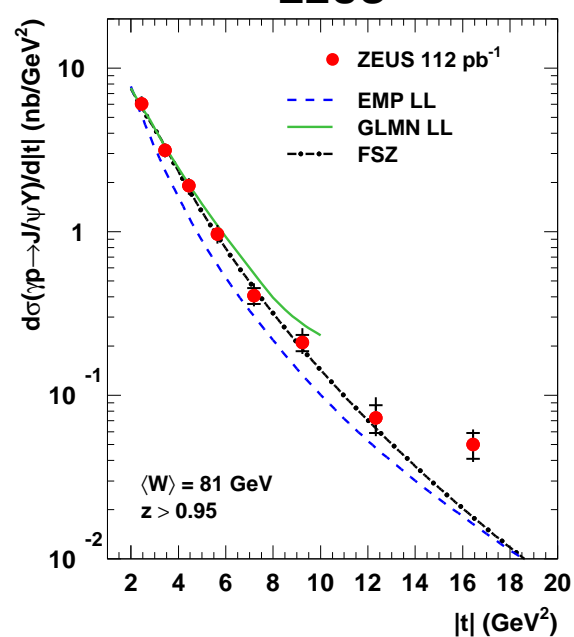

\section{ZEUS}

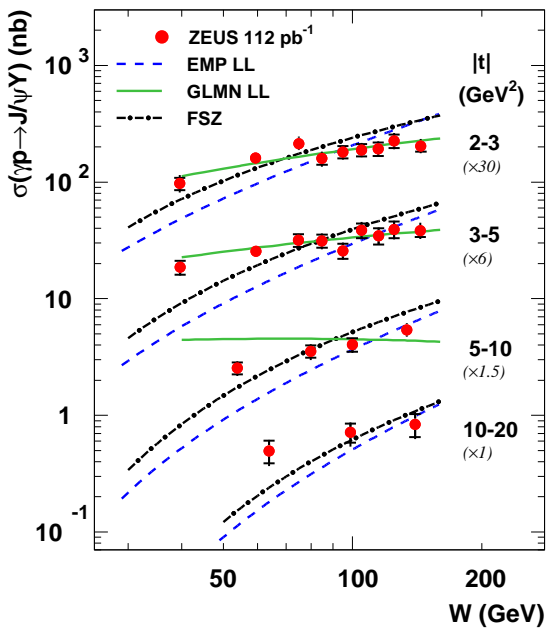

Figure 3: The differential cross-section, $d \sigma / d|t|$, (left) and the $W$ dependence (right) for the process $\gamma p \rightarrow$ $J / \psi Y$. The lines show the predictions of the calculations described in the text.
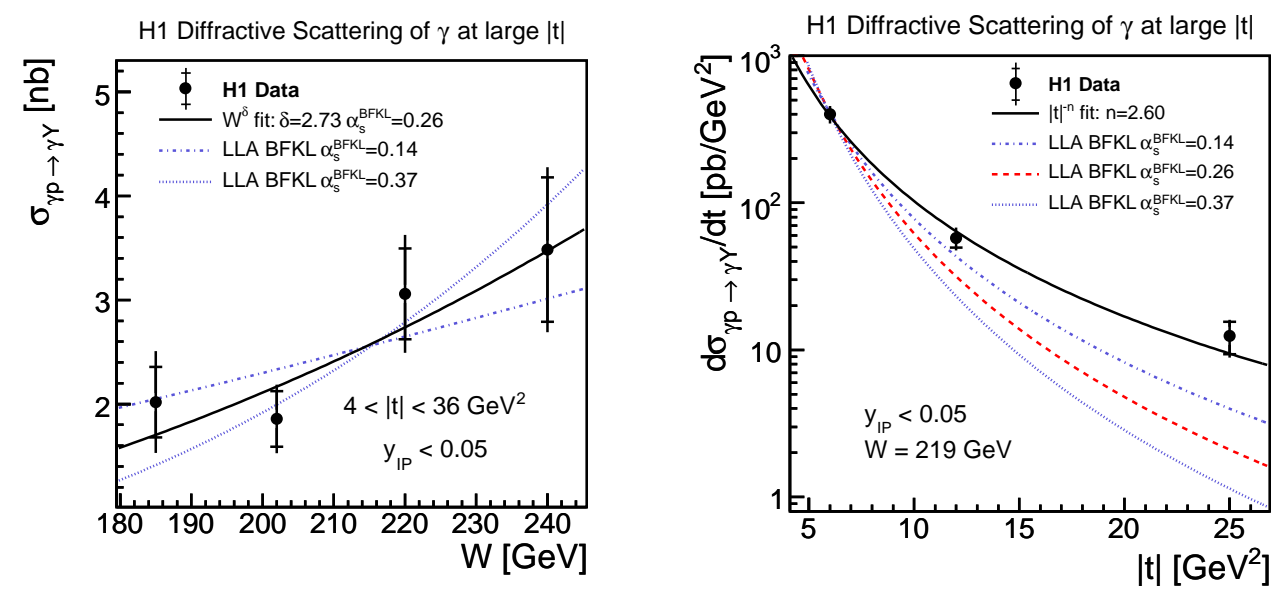

Figure 4: The $W$ dependence (left) and the differential cross-section, $d \sigma / d|t|$, (right) for the process $\gamma p \rightarrow$ $\gamma Y$. The lines show the predictions of the calculations described in the text.

approximation of the BFKL model the cross section is proportional to $\sigma(W) \sim W^{4 \omega_{0}}$, where the exponent is given by the choice of $\alpha_{S}^{B F K L}$ by $\omega_{0}=\left(3 \alpha_{S}^{B F K L} / \pi\right) 4 \ln 2$. Using the $\delta$ value measured from the $W$ dependence of the cross section the value $\alpha_{S}^{f i t}=0.26 \pm 0.10$ (stat. $)_{-0.07}^{+0.05}$ (syst.) was extracted. The other predictions were calculated for $\alpha_{S}^{B F K L}=0.14$ and 0.37 corresponding to one standard deviation of $\alpha_{S}^{f i t}$. The $W$ cross section within the errors is well described by the LLA prediction, while in the case of the $d \sigma / d t$ the BFKL calculations are to steep for all three values of $\alpha_{S}^{B F K L}$.

\section{References}

[1] G. Wolf, arXiv:0907.1217v1 [hep-ex] 
[2] S.J. Brodsky et al., Phys. Rev. D 50 (1994) 3134

[3] P.D.B. Collins, An Introduction to Regge Theory and High Energy Physics, Cambridge University Press, 1977

[4] ZEUS Coll., S. Chekanov et al., Phys. Lett. B (Ref. No. PLB26003)

[5] ZEUS Coll., J. Breitweg et al., Phys. Lett. B 437 (1998) 432

[6] H1 Coll., C. Adloff et al., Phys. Lett. B 483 (2000) 23

[7] L.L. Frankfurt, M.F. McDermott and M. Strikman, JHEP 9902 (1999) 002

[8] D.Yu. Ivanov, G. Krasnikov and L. Szymanowski, Nucl. Phys. B (Proc. Suppl.) 146 (2005) 134

[9] A.D. Martin et al., Phys. Lett. B 662 (2008) 252

[10] A. Rybarska, W. Schäfer and A. Szczurek, Phys. Lett. B 668 (2008) 126

[11] ZEUS Coll., S. Chekanov et al., to be published in JHEP

[12] A. Donnachie and P.V. Landshoff, Phys. Lett. B 296 (1992) 227

[13] S.J. Brodsky et al., JETP Lett. 70 (1999) 155

[14] N.N. Nikolaev, B.G. Zakharov and V.R. Zoller, Phys. Lett. B 366 (1996) 337

[15] A. Donnachie and P.V. Landshoff, Nucl. Phys. B 231 (1984) 189

[16] E. Gotsman et al., Phys. Lett. B 532 (2002) 37

[17] R. Enberg, L. Motyka and G. Poludniowski, Eur. Phys. J. C 26 (2003) 219

[18] L. Frankfurt, M. Strikman and M. Zhalov, Phys. Lett. B 670 (2008) 32

[19] L. Frankfurt and M. Strikman, Phys. Rev. Lett. 63 (1989) 1914

[20] H1 Coll., DIS 2009, April 26-30, 2009, Madrid, H1prelim-09-017 (http://www-h1.desy.de/publications/H1preliminary.short_list.html)

[21] H1 Coll., F.D. Aaron et al., Phys. Lett. B 672 (2009) 219

[22] D. Y. Ivanov and M. Wüsthoff, Eur. Phys. J. C 8 (1999) 107

[23] N. G. Evanson and J. R. Forshaw, Phys. Rev. D 60 (1999) 034016

[24] B. E. Cox and J. R. Forshaw, J. Phys. G 26 (2000) 702 